\title{
THE SPATIAL REPRODUCTIVE VALUE AND THE SPATIAL MOMENTUM OF ZERO POPULATION GROWTH
}

\author{
Andrei Rogers \\ Frans Willekens
}

December 1976

Research Memoranda are interim reports on research being conducted by the International Institute for Applied Systems Analysis, and as such receive only limited scientifir review. Vicws or opinions contained herein do not necessarily represent those of the Institute or of the National Member Organizations supporting the Institute.

This paper is a completely revised version of "Spatial zero Population Growth," which was presented at the Annual Meeting of the Population Association of America, April 29 - May 1, 1976, Montreal, Canada. 


\section{Preface}

Interest in human settlement systems and policies has been a critical part of urban-related work at IIASA since its inception. Recently this interest has given rise to a concentrated research effort focusing on migration dynamics and settlement patterns. Four sub-tasks form the core of this research effort:

I. the study of spatial population dynamics;

II. the definition and elaboration of a new research area called demometrics and its application to migration analysis and spatial population forecasting;

III. the analysis and design of migration and settlement policy;

IV. a comparative study of national migration and settlement patterns and policies.

This paper, the ninth in the dynamics series, explores some of the redistributional consequences of zero growth. It develops the concept of the spatial reproductive value and demonstrates how this measure can be used to assess the quantitative impacts of fertility reduction.

Related papers in the dynamics series, and other publications of the migration and settlement study, are listed on the back page of this report.

Andrei Rogers

December 1976 


\section{Abstract}

This paper develops the concept of spatial reproductive value and illustrates how such a notion may be used to trace out quantitatively the geographical impacts of a sudden reduction in fertility to bare replacement level. Such a reduction does not immediately produce zero population growth in populations that previously experienced high birth rates. The built-in momentum for continued growth and its spatial dimension may be assessed with the aid of spatial reproductive values. 


\section{Table of Contents}

1. Introduction

2. Spatial Zero Population Growth

2.1 Conditions for Spatial Zero

Population Growth

2.2 The Redistributional Impacts of Two

Alternative Spatial Patterns of Fertility Reduction

3. The Spatial Reproductive Value

3.1 Definitions and Theory

3.2 Numerical Evaluation

3.3 Stable Population Analysis

4. The Spatial Momentum of Zero Population Growth

4.1 The Ultimate Size and Distribution of a Stationary Spatial Population

4.2 The Spatial Momentum of an Initially stable Population

4.3 The Urbanization Momentum in India

5. Conclusion 

The Spatial Reproductive Value and the Spatial

Momentum of Zero Population Growth

\section{Introduction}

If age-specific birth and death rates remain fixed, a population that is undisturbed by migration will ultimately evolve into a stable population that increases at a fixed annual rate, $r$ say. If the birth rates are fixed at bare replacement level, then such a stable population will be a stationary or zerogrowth population with an $r$ of zero. In what follows, we explore some of the spatial consequences of zero growth by considering how a sudden reduction of fertility to replacement level might affect the spatial evolution of a multiregional population whose constituent regional populations experience the redistributional effects of internal migration.

Mathematical analysis of spatial zero population growth (SZPG) can be facilitated by adopting a spatial generalization of the notion of reproductive value first set out by fisher (1929, p. 27). Keyfitz (1975) has shown how the quantitative impacts of fertility reduction, of sterilization, of mortality, and of emigration, all assumed to take place at a particular age $\mathrm{x}$ within a single population, can be assessed by means of reproductive values. Analogous calculations can be carried out for multiple spatially interacting populations with the aid of spatial reproductive values, as we demonstrate for the case of fertility reduction to replacement level.

Finally, it is well-known that a sudien reduction of fertility to replacement level does not immediately produce zero population growth. Children outnumber parents in a population that maintains high birth rates. Consequently the number of potential parents in the next generation will inevitably be larger than at present, and the current population therefore has a built-in tendency to continue growing before it ultimately stabilizes into a zero-growth condition. This built-in momentum may be assessed with the aid of reproductive values, and the 
concluding parts of this paper illustrate how such a momentum may be accorded a spatial dimension.

\section{Spatial Zero Population Growth}

Zero population growth for a nation implies a zero growth average for local areas. This, of course, still allows for the possibility of nonzero growth in particular localities. Thus spatial zero growth, like temporal zero growth, may be viewed either as a condition that ultimately prevails uniformly over space and time, or one that exists only because of a fortuitous balancing of regional rates of positive growth, of zero growth, and of decline. Since no obvious advantages arise from the latter case, it is quite natural to view the attainment of temporal zero growth in the long-run in terms of an indefinite continuation of temporal zero growth in the short-run. We follow this tradition in this paper and view the attainment of spatial zero growth in the long-run in terms of temporal zero growth within each region of a closed multiregional population system. Consequently, we confine our attention to the evolution of a particular subset of stationary populations, called spatial zero growth populations, i.e., stable multiregional populations that have a zero growth rate. Thus we augment the usual twin assumptions of a fixed mortality schedule and a fixed fertility schedule, set at replacement level, with the assumption of a fixed migration schedule. Multiregional populations subjected to such regional growth regimes ultimately assume a persisting zero rate of growth in every region and exhibit zero growth both over time and over space.

\subsection{Conditions for Spatial Zero Population Growth}

Stable population theory informs us that a stationary, say female, population arises out of the combination of the following conditions [Ryder, 1974]: (i) a fixed survival function $\ell(a)$ which specifies the probability that a female survives to age $a_{;}$(ii) a fixed maternity function $m(a)$ which defines the 
conditional probability that a surviving female gives birth to a baby girl at age a; (iii) a product-sum of these two functions, the net reproduction rate

$$
R(0)=\int_{0}^{\infty} \ell(a) m(a) d a,
$$

that is equal to unity; and (iv) an absence of migration.

If $l(a)$ and $m(a)$ are fixed for a long time, and the population is closed to migration, the annual number of female births, $B(t)$, is given by the well-known Lotka equation

$$
B(t)=\int_{0}^{\infty} B(t-a) \quad \ell(a) m(a) d a .
$$

Substituting $Q e^{r t}$ for $B(t)$ in the above equation gives

$$
Q=Q\left[\int_{0}^{\infty} e^{-r a} \ell(a) m(a) d a\right]=Q \Psi(r)
$$

whose solution is that value of $r$ for which $\Psi(r)=1$, and where $Q$ denotes the number of stable equivalent births.

For the special case of $r=0, B(t)=\hat{Q}$, and

$$
\hat{\mathrm{Q}}=\hat{\mathrm{Q}}\left[\int_{0}^{\infty} \ell(\mathrm{a}) \hat{\mathrm{In}}(\mathrm{a}) \mathrm{da}\right]=\hat{\mathrm{Q}} \hat{\mathrm{R}}(0),
$$

where $\hat{\mathrm{m}}(\mathrm{a})=\gamma \mathrm{m}(\mathrm{a})$ and $\gamma$ is generally taken to be equal to the reciprocal of the net reproduction rate, $R(0)$. (The caret will henceforth be used to distinguish the parameters of a stationary population.)

Let us now relax the last of the four conditions that generate a stationary population, i.e., closure of the population to migration. Imagine a multiregional population whose 
long-run evolution follows the multiregional Lotka equation (Rogers, 1975, Ch. 4):

$$
\left.\{\underset{\sim}{B}(t)\}=\int_{0}^{\infty} \underset{\sim}{m}(a) \underset{\sim}{\ell}(a) \underset{\sim}{\{}(t-a)\right\} d a,
$$

where $\underset{\sim}{m}(a)$ is a diagonal matrix of annual regional fertility rates $m_{j}(a), \underset{\sim}{\ell(a)}$ is a matrix of place-of-birth to placeof-residence survival probabilities $i_{j}^{l}(a)$, and $\{\underset{\sim}{B}(t)\}$ is $a$ vector of regional births $B_{j}(t)$.

Substituting $\{\underset{\sim}{O}\} e^{r t}$ for $\{\underset{\sim}{B}(t)\}$ in (1) gives

$$
\{\underset{\sim}{Q}\}=\left[\int_{0}^{\infty} \mathrm{e}^{-r a} \underset{\sim}{\mathrm{m}}(\mathrm{a}) \underset{\sim}{\ell}(\mathrm{a}) \mathrm{da}\right]\{\underset{\sim}{\underline{\sim}}\}=\underset{\sim}{\Psi}(r)\{\underset{\sim}{Q}\},
$$

whose solution is that value of $r$ for which the dominant characteristic root of $\Psi(r)$ is unity. For the special case of $r=0$, $\{\underset{\sim}{B}(t)\}=\{\underline{\hat{Q}}\}$, and

$$
\{\underset{\sim}{\hat{Q}}\}=\left[\int_{0}^{\infty} \hat{\sim} \underset{\sim}{\hat{m}}(a) \underset{\sim}{\ell}(a) d a\right]\{\underset{\sim}{\hat{Q}}\}=\underset{\sim}{\hat{R}}(0)\{\underset{\sim}{\hat{Q}}\},
$$

where carets are once again used to distinguish stationary population measures. The element in the $i^{\text {th }}$ row and the $j^{\text {th }}$ column of $\underset{\sim}{\hat{R}}(0)$ is the stationary regional net reproduction rate in region $i$ of women born in region $j$ :

$$
j^{\hat{R}_{i}}(0)=\int_{0}^{\infty} j^{\ell}(a) \hat{m}_{i}(a) d a .
$$

Equation (3) shows that for a spatial zero growth population to be maintained, the dominant characteristic root of the matrix $\underset{\sim}{\hat{R}(0)}$ must be unity. Consequently a reduction of fertility to replacement level may be interpreted as a reduction of the 
elements of $\mathrm{m}(\mathrm{a})$ to a level that reduces the dominant characteristic root of a given net reproduction matrix $R(0)$ to unity. Such an operation transforms $\underset{\sim}{m}(a)$ to $\underset{\sim}{\hat{m}}(a)$ and $\underset{\sim}{R}(0)$ to $\underset{\sim}{\hat{R}}(0)$.

The vector $\{\hat{Q}\}$ in (3) is the characteristic vector associated with the unit dominant characteristic root of $\underset{\sim}{\hat{R}}(0)$ and denotes the total number of births in each region of a spatial zero growth population. The proportional allocation of total births that it defines is directly dependent on the transformation that is applied to change $\underset{\sim}{R}(0)$ to $\underset{\sim}{\hat{R}}(0)$. Since in a spatial zero growth population the regional stationary equivalent population $\hat{Y}_{i}$ is equal to the guotient formed by $\hat{Q}_{i}$ and the birth rate $\hat{b}_{i}$, we see that the aifferent ways in which $\underset{\sim}{R}(0)$ is transformed into $\underset{\sim}{\mathrm{R}}(0)$ become, in fact, alternative "spatial paths" to a stationary multiregional population.

2.2 The Redistributional Impacts of Two Alternative Spatial Patterns of Fertility Reduction

A multiregional population system that is growing at a positive rate of growth exhibits a net reproduction matrix $\underset{\sim}{R}(0)$ with a dominant characteristic root $\lambda_{1}[\underset{\sim}{R}(0)]$ that is greater than unity. If the rate of childbearing in each region of this population system were immediately modified such that every woman born in that region would now have a net reproduction rate of unity, then

$$
{ }_{i} \hat{R}(0)=\sum_{j=1}^{m} i_{j} \hat{R}_{j}(0)=1
$$

or, in matrix form,

$$
\underset{\sim}{\hat{R}}(0)^{\prime}\{\underset{\sim}{\{1\}}=\underset{\sim}{\{1\}},
$$

where the prime denotes transposition.

Following the normal practice in single-region exercises of this kind, assume that the fertility of each regional cohort 
of women is modified through the multiplication of each region's age-specific birth rates by a fixed fertility adjustment factor, $\gamma_{i}$ say. Then

$$
i_{i} \hat{R}(0)=\sum_{j=1}^{m} i^{R_{j}}(0)=\sum_{j=1}^{m} \int^{\infty} i_{j}^{l}(a) \gamma_{j} m_{j}(a) d a=\sum_{j=1}^{m} \gamma_{j} i_{j}(0)=1
$$

and

$$
\hat{\sim}(0)=\underset{\sim}{\gamma} R(0),
$$

where $\underset{\sim}{\gamma}$ is a diagonal matrix of fertility adjustment factors. Substituting (5) into (4) gives

$$
\underset{\sim}{R}(0){\underset{\sim}{Y}}_{\sim}\{\underset{\sim}{1}\}=\{\underset{\sim}{1}\},
$$

whence

$$
\left.\{\underset{\sim}{\underline{\gamma}}\}=\left[\underset{\sim}{R}(0)^{\prime}\right]^{-1} \underset{\sim}{\{1}\right\}
$$

Setting the fertility of each female cohort in every region to bare replacement level, the cohort replacement alternative, is but one of many possible spatial patterns of fertility reduction. One could instead, for example, consider a fertility reduction scheme in which the aggregate system-wide net reproduction rate is reduced to unity through the multiplication of all age-specific birth rates by the same fertility adjustment factor, $\gamma$ say. That is, let

$$
\begin{aligned}
\underset{\sim}{\hat{R}}(0) & =\underset{\sim}{\gamma \underset{\sim}{R}(0),} \quad \text { where } \\
\gamma & =\frac{1}{\lambda_{1}[\underset{\sim}{R}(0)]} .
\end{aligned}
$$

This particular spatial pattern of fertility reduction may be called the proportional reduction alternative, and its redistributional impacts can he quite different. 
A numerical illustration may be instructive at this point. Assume that the net reproduction behavior of the urban and rural female populations of a national population are approximated by the net reproduction matrix

$$
\underset{\sim}{R}(0)=\left[\begin{array}{cc}
u^{R_{u}(0)} & r^{R_{u}(0)} \\
u^{R_{r}(0)} & r^{R_{r}(0)}
\end{array}\right]=\left[\begin{array}{cc}
3 / 4 & 1 / 2 \\
1 / 4 & 1
\end{array}\right]
$$

where, for example, $r_{u}(0)=1 / 2$ denotes the net reproduction rate in urban areas of rural-born women. In other words, under the observed regime of growth, each woman born in rural areas will, on the average, replace herself in the succeeding generation by $11 / 2$ daughters, one third of whom will be born in urban areas. Urban-born women, on the other hand, have a lower net reproduction rate, i.e., ${ }_{u} R(0)=1<r^{R(0)}$, which when combined with the net reproduction rate of rural-born women gives the national female population an overall net reproduction rate of $\lambda_{1}[\underset{\sim}{R}(0)]=11 / 4$, where $\lambda_{1}[\underset{\sim}{R}(0)]$ is the dominant characteristic root of the net reproduction matrix in (8).

An immediate system-wide fertility decline to replacement level, such that each urban- or rural-born woman is followed in the next generation by exactly one daughter, implies $\gamma_{\mathrm{u}}=11 / 5$ and $\gamma_{\mathrm{r}}=2 / 5$, whence

$$
\underset{\sim}{\hat{R}}(0)=\left[\begin{array}{ll}
9 / 10 & 3 / 5 \\
1 / 10 & 2 / 5
\end{array}\right] \text { and }\{\underset{\sim}{\hat{Q}}\}=\left[\begin{array}{l}
6 \\
1
\end{array}\right] \text {. }
$$

Both groups of women now exhibit unit rates of net reproduction; the dominant characteristic root of $\underset{\sim}{\hat{R}}(0)$ is unity; and the characteristic vector associated with the unit dominant characteristic root of $\underset{\sim}{\hat{R}}(0)$ indicates that $6 / 7$ of the total births in the spatial zero growth population will occur in urban areas. Consider next the redistributional implications of a fertility decline according to the proportional reduction 
alternative defined in (7). In this instance, $\gamma=4 / 5$, whence

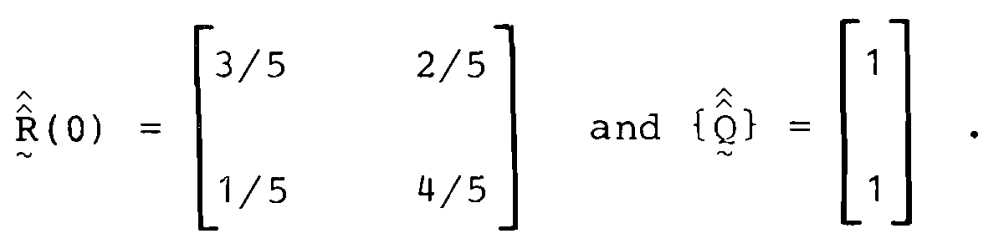

The fertility of urban-born women now declines to below replacement level $\left(\mathrm{u}_{\mathrm{u}}(0)=4 / 5\right)$, whereas that of rural-born women continues to exceed such a level $\left({ }_{r} \hat{\hat{R}}(0)=11 / 5\right)$. The dominant characteristic root of $\underset{\sim}{\hat{R}}(0)$ is unity, and urban and rural areas have an equal number of births.

\section{The Spatial Reproductive Value}

The concept of reproductive value, as developed by R.A. Fisher (1929), revolves around the notion of regarding the offspring of a child as the repayment of a debt. Specifically, if the birth of a baby is viewed as a loan of a life and if the future offspring of this child are viewed as the subsequent repayment of this loan, suitably discounted at the annual rate $r$ and compounded momently, then the present value of the repayment may be taken to be

$$
\int_{0}^{\infty} e^{-r a} \ell(a) m(a) d a .
$$

Equating the loan with the discounted repayment gives

$$
1=\int_{0}^{\infty} e^{-r a} \ell(a) m(a) d a,
$$

which is recognizable as the characteristic equation used to solve for $r$, the intrinsic rate of growth. Thus, as Keyfitz points out:

"the equation can now be seen in a new light: the equating of loan and discounted repayment is 
what determines $r, r$ being interpretable either as the rate of interest of an average loan or as Lotka's intrinsic rate of natural increase."

(Keyfitz, 1975, p. 588)

In the above cited paper, Keyfitz considers how much of the debt is outstanding by the time the child has reached age $x$. He defines this quantity to be $v(x)$, the reproductive value at age $\mathrm{x}$, where

$$
v(x)=\int_{x}^{\infty} e^{-r(a-x)} \frac{\ell(a)}{\ell(x)} m(a) d a
$$

and $v(0)$ is scaled to equal unity.

\subsection{Definitions and Theory}

Keyfitz's arguments have their spatial (multiregional) counterparts. To develop these it is convenient to re-express (9) for arbitrary values of $v(0)$, namely:

$$
v(x)=v(0) \int_{x}^{\infty} e^{-r(a-x)} \ell(a) \ell(x)^{-1} m(a) d a=v(0) n(x)
$$

where

$$
v(0)=v(0) \int_{0}^{\infty} e^{-r a} \ell(a) m(a) d a=v(0) \psi(r)
$$

and $\mathrm{n}(\mathrm{x})$ denotes the total discounted number of baby girls expected to be born to a woman now at age $x$. This form of the equation immediately suggests the multiregional analog

$$
\begin{aligned}
& \{\underset{\sim}{v}(x)\}^{\prime}=\{\underset{\sim}{v}(0)\}^{\prime} \int_{x}^{\infty} e^{-r(a-x)} \underset{\sim}{m}(a) \underset{\sim}{\ell}(a) \underset{\sim}{\ell}(x)^{-1} d a, \\
& =\{v(0)\}^{\prime} n(x), \text { say, }
\end{aligned}
$$


where

$$
\{\underset{\sim}{v}(0)\}^{\prime}=\{\underset{\sim}{v}(0)\}^{\prime} \int_{0}^{\infty} e^{-r a} \underset{\sim}{m}(a) \underset{\sim}{\ell}(a) d a=\{\underset{\sim}{v}(0)\}^{\prime} \underset{\sim}{\Psi}(r)
$$

The matrix $\mathrm{n}(\mathrm{x})$ represents the expected total number of female offspring per woman at age $x$, discounted back to age $x$. The element $n_{i j}(x)$ gives the discounted nurber of female children to be born in region $j$ to a woman now $x$ years of age and a resident of region $i$. The vector $\{\underset{\sim}{v}(x)\}$ represents the reproductive values of $x$-year old women, differentiated by region of residence. Observe that the elements of $\{\mathrm{v}(\mathrm{x})\}$ depend on the scaling given to $\{\underset{\sim}{v}(0)\}$, the left characteristic vector associated with the unit dominant characteristic root of the characteristic matrix $\underset{\sim}{\Psi}(r) .1$ Thus in the multiregional model, the reproductive value of a baby girl depends on where she is born.

Equations (10) through (12) may be given the following demographic interpretation. If lives are loaned to regions according to the (column) vector $\{\underset{\sim}{Q}\}$ then the amount of "debt" outstanding $x$ years later is given by the (row) vector $\{\underset{\sim}{v}(x)\}^{\prime}$, the regional expected values of subsequent offspring discounted back to age $x$. The elements of this vector, therefore, may be viewed as spatial. (regional) reproductive values at age $\mathrm{x}$.

A slightly modified perspective of the spatial reproductive value is adopted in this paper. Specifically, we shall distinguish between the terms number and value when referring to births in the various regions of a closed multiregional system. The two expressions have identical meanings in the single-region model, but variations in regional fertility and mortality schedules give them different meanings in any multiregional model in which internal migration is represented.

${ }^{1}$ Recall that in (2) the corresponding right characteristic vector of $\underset{\sim}{\Psi}(r)$ was $\{\underset{\sim}{Q}\}$. 
Recall the definition of the multiregional characteristic matrix $\underset{\sim}{\Psi}(r)$ in Equation (2). An element ${ }_{i} \Psi_{j}(r)$ denotes the discounted total number of daughters born in region $j$ to a mother born in region $i$. The discounted number of female births per woman in a particular region, $i^{\Psi} \cdot(r)=\sum_{j} i^{\Psi}{ }_{j}(r)$, may be less than unity. Although this suggests that she does not repay the full amount of her "debt" to society, we shall show that this may be true only of number but not of value.

Consider society as an investor in a multiregional (spatial) portfolio of lives, each element of the portfolio being a region. The society distributes its investment in lives among its regions according to the vector $\{\underset{\sim}{Q}\}$. The total discounted number of offspring attributable to the $Q_{i} i$-born individuals is

$$
i^{\Psi} \cdot Q_{i}=\sum_{j} i_{j}^{\Psi} Q_{i}
$$

out of which total ${ }_{i}{ }_{j} Q_{i}$ will be born in region $j$. The total societal investment in region $i$, however, is $Q_{i}$ births, which by virtue of Equation (2) may be expressed as

$$
Q_{i}=\sum_{j} j_{i}^{\Psi}(r) Q_{j}
$$

Hence for each region, during stable growth, the present discounted number of future births in that region must be equal to the current number of births.

The distribution of total societal births among regions is one of two related aspects of the reproductive value problem. Associated with this primal aspect of allucation is a dual aspect of valuation. ${ }^{2}$

2 This primal-dual relationship resembles the one found in mathematical programming theory and suggests the conjecture that (i) the primal optimization problem is one of selecting an allocation of births to maximize growth, within the constraints of a given regime of fertility, mortality, and migration; and (ii) the dual optimization problem is one of valuing births in each region so as to minimize the total regionallyweighted societal reproductive value. 
The value of a birth reflects the capacity to produce new life. If a 0-year old in region $i$ is worth $v_{i}(0)$, then, by Equation (11), the reproductive value of the discounted number of offspring must also be $v_{i}(0)$, that is,

$$
v_{i}(0)=\sum_{j} v_{j}(0) i_{j}^{\Psi}(r)
$$

Thus, if the investment in one life in a region is viewed as a debt of an individual to society, then in a stable equilibrium each individual must repay that debt to society at an annual interest rate $r$. The repayment does not have to take place in the region of birth, however. Part of it can occur in other regions, where births may be worth more (or less) than in the region of birth. Thus we may conclude that individuals pay back their debt to society in values $\mathrm{v}(0)$, whereas regions pay back their debt in numbers $Q$. The former distribution is defined by (12); the latter derives from (2).

Spatial reproductive values at age $x, v_{i}(x)$, may be appropriately consolidated to yield total spatial reproductive values, $v_{i}$, by means of the relationship

$$
\begin{aligned}
\{\underset{\sim}{v}\}^{\prime} & =\int_{0}^{\infty}\{\underset{\sim}{v}(x)\}^{\prime} \underset{\sim}{k}(x) d x \\
& =\{\underset{\sim}{v}(0)\}^{\prime}\left[\int_{0}^{\infty} \underset{\sim}{n}(x) \underset{\sim}{k}(x) d x\right] \\
& =\{\underset{\sim}{v}(0)\}^{\prime} \underset{\sim}{n},
\end{aligned}
$$

where $\underset{\sim}{\mathrm{k}}(\mathrm{x})$ is a diagonal matrix with $\mathrm{k}_{i i}(\mathrm{x})$ representing the number of women at age $x$ in region $i$, and $\underset{\sim}{n}$ is a matrix of total discounted number of female offspring associated with that population. The total reproductive value of the multiregional population then is

$$
v=\{\underset{\sim}{v}\}^{\prime}\left\{\underline{\sim}_{1}\right\}
$$




\subsection{Numerical Evaluation}

The definition of $\{\underset{\sim}{\mathrm{v}}(\mathrm{x})\}$ in $(10)$ refers to exact age $\mathrm{x}$. A suitable numerical approximation, analogous to the one usually made in the single-region model, is

$$
\{\underset{\sim}{v}(x)\}^{\prime}=\{\underset{\sim}{v}(0)\}^{\prime} \sum_{a=x}^{\beta-5}\left[e^{-r(a+2 \cdot 5-x)} \underset{\sim}{M}(a) \underset{\sim}{L}(a)\right]{\underset{\sim}{l}}^{-1}(x)
$$

where $\beta$ is the last age of childbearing, $\underset{\sim}{\mathrm{M}}(\mathrm{a})$ and $\underset{\sim}{\mathrm{L}}(\mathrm{a})$ are, respectively, matrices of fertility rates and life table populations of women aged a to $a+4$, and $\underset{\sim}{\ell}(x)$ is the life-table survival matrix to exact age $x$ (see Rogers, 1975).

The average spatial reproductive value for the age interval $\mathrm{x}$ to $\mathrm{x}+4$ at last birthday is denoted by $\{\underset{\sim}{\mathrm{V}}(\mathrm{x})\}^{\prime}$ and may be approximated by

$$
\begin{aligned}
& \{\underset{\sim}{V}(x)\}^{\prime}=\{\underset{\sim}{v}(0)\}^{\prime} \frac{5}{2} \sum_{a=x}^{\beta-5}\left[e^{-r(a-x)} \underset{\sim}{M}(a) \underset{\sim}{L}(a)\right. \\
& \left.+e^{-r(a+5-x)} \underset{\sim}{M}(a+5) \underset{\sim}{L}(a+5)\right]{\underset{\sim}{L}}^{-1}(x) \\
& =\{\underset{\sim}{V}(0)\}^{\prime} \underset{\sim}{N}(x),
\end{aligned}
$$

where $\underset{\sim}{N}(x)$ is the average value of $\underset{\sim}{n}(x)$ for the age interval $x$ to $x^{2}+4$ at last birthday. 3

Table 1 presents the values of $\underset{\sim}{N}(x)$ for $x=0,5, \ldots, 50$ using the 1961 population data on Yugoslavian females that is published in Rogers (1975). For example, the discounted $(r=0.006099)$ number of daughters expected to be born to a woman now living in slovenia and 15 to 19 years old is 1.0078 . of this total 0.9417 will be born in slovenia and 0.0661 will be born in the rest of Yugoslavia. A woman in the same age

3 Equation (17) may be shown to be consistent with a somewhat different formulation set out as Equation (4.37) on p. 105 of Rogers, 1975. 
group in the rest of Yugoslavia has an expected discounted number of daughters of 1.1943, of which only 0.0068 will be born in slovenia. This is mainly a reflection of the low level of migration from the rest of Yugoslavia to Slovenia and the low fertility in slovenia.

The characteristic matrix for this two-region system is

$$
\underset{\sim}{\Psi(0.006099)}=\left[\begin{array}{ll}
0.813587 & 0.009081 \\
0.103594 & 0.994840
\end{array}\right] ;
$$

its dominant characteristic root is unity; and the associated left and right characteristic vectors are, respectively:

$$
\{\underset{\sim}{v}(0)\}^{\prime}=\left[\begin{array}{ll}
1 & 1.798369
\end{array}\right] \quad\{\underset{\sim}{Q}\}=\left[\begin{array}{l}
1 \\
20.515385
\end{array}\right] \text {. }
$$

Note that the discounted number of female offspring of a baby girl born in slovenia is less than unity. Nevertheless she still repays her debt of a life to society, because the 0.104 daughters born to her in the rest of Yugoslavia have a higher value than an equivalent number born in slovenia. The weighted discounted repayment is a single life:

$$
1=1(0.813587)+1.798369(0.103594)
$$

Combining the numerical approximation of $\underset{\sim}{N}(x)$ with that of $\{v(0)\}^{\prime}$, as set out in (17), gives the set of values for $\{\underset{\sim}{\mathrm{V}}(\mathrm{x})\}^{\prime}$ in Table 2. These indicate that the spatial reproductive values of slovenian girls are, at most ages, roughly half of the corresponding values for girls living in the rest of Yugoslavia. 
Finally, weighting the age-specific values of $\underset{\sim}{N}(x)$ in Table 1 by the respective observed populations and adding gives the total discounted number of female offspring $\underset{\sim}{N}$. Table 3 shows that under the 1961 regime of fertility, mortality, and migration, the total discounted number of daughters to be born to Yugoslavia's 1961 female population is 5,528,742.4 of these, 383,133 or 6.93 percent will be born in slovenia, and 379,208 or 6.86 percent will be children of the observed 1961 female residents of slovenia. Of the ultimate discounted 383,133 female births in slovenia, 30,404 can be attributed to women now residing in the rest of Yugoslavia and 352,729 to potential mothers now living in slovenia.

To derive the total reproductive value of the observed female population one must weight the discounted number of offspring according to region of birth. If we assign a value of unity to a birth in Slovenia then 1.798369 is the corresponding value of a birth in the rest of Yugoslavia. The total reproductive value of Slovenian women is (Table 3)

$$
352,729(1)+26,479(1.798369)=400,347
$$

and the corresponding value for women residing in the rest of Yugoslavia is

$$
30,404(1)+5,119,130(1.798369)=9,236,491 .
$$

Adding the two subtotals together gives the aggregate systemwide total reproductive value

$$
V=400,347+9,236,491=9,636,838,
$$

for the case where $v_{1}(0)$ is set equal to unity.

4 The slight discrepancy between this total and the one reported on p. 114 of Rogers (1975) may be attributed to differences in computer hardware. 


\subsection{Stable Population Analysis}

The reproductive value may be used to establish the extent to which an individual of a given age will, on the average, contribute to the births of future generations. If $k$ is the mean age of childbearing in a stable population then

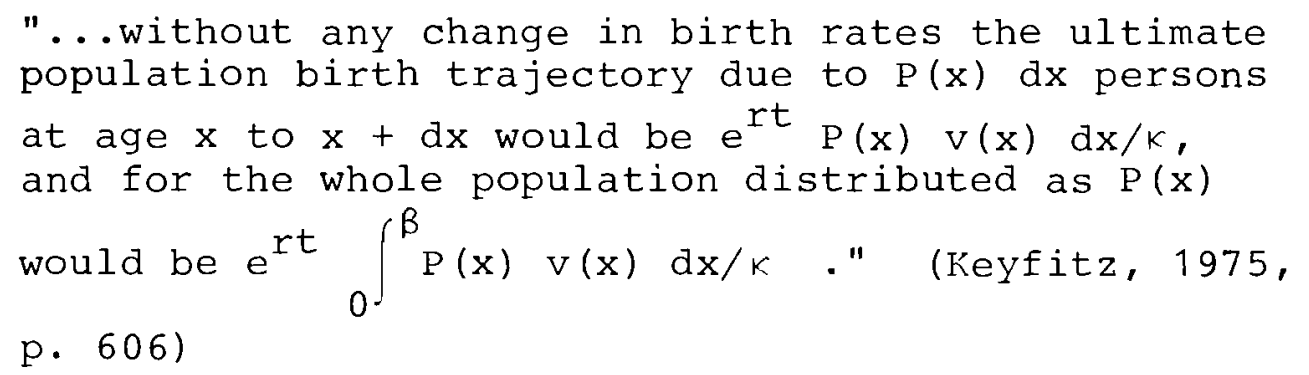

The above holds true for a single-region population that is closed to migration. It can be shown that the corresponding result for a multiregional population system is (Willekens, 1977):

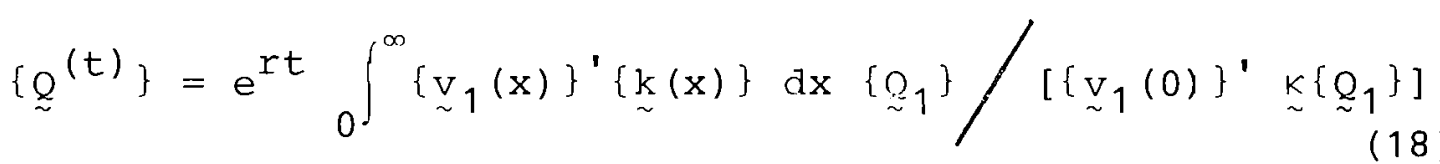

where we denote the initial population by $k(x)$ instead of $P(x)$, to maintain consistency with our earlier notation; where the unit subscripts designate vectors with arbitrary scalings (we shall adopt a scaling that sums the elements of each vector to unity); and where $\underset{\sim}{k}$, the matrix of mean ages of childbearing in the stable population, is defined as

$$
\underset{\sim}{K}=\underset{\sim}{R}(r)(1)[\underset{\sim}{R}(r)(0)]^{-1},
$$

with

$$
\underset{\sim}{\mathrm{R}}(\mathrm{r})(\mathrm{p})=\int_{0}^{\infty} \mathrm{x}^{\mathrm{p}} \mathrm{e}^{-\mathrm{rx}} \underset{\sim}{\mathrm{m}}(\mathrm{x}) \underset{\sim}{\ell}(\mathrm{x}) \mathrm{dx}, \mathrm{p}=0,1 .
$$


Rewriting (18) as

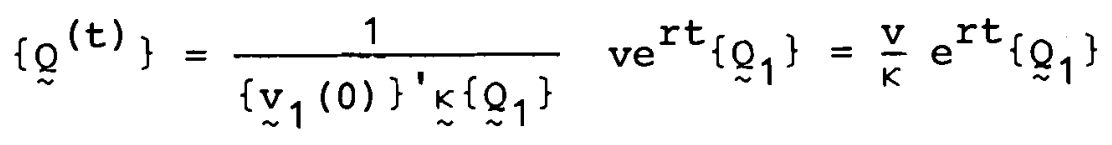

we immediately obtain an expression for stable equivalent births :

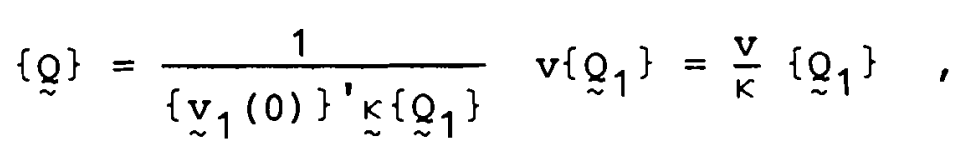

where

$$
K=\left\{{\underset{\sim}{v}}_{1}(0)\right\}^{\prime} \underset{\sim}{K}\left\{{\underset{\sim}{Q}}_{1}\right\}
$$

Goodman (1969) proves that the impact on the ultimate stable birth trajectory of a girl at age $x$ is $v(x) e^{r t} / \kappa$. The above results indicate that the size of this impact depends on the spatial reproductive value of the girl and, therefore, on her initial region of residence. The spatial proportional distribution of these births, however, is independent of the initial distribution of the population and is therefore not a function of her initial region of residence.

The ultimate birth trajectory, as given by (18), depends on the intrinsic annual rate of growth $r$, the total reproductive value of the population $v$ and its proportional distribution among the regions $\left\{\underline{\sim}_{1}(0)\right\}^{\prime}$, the matrix of mean ages of childbearing in the stable population $\underset{\sim}{k}$, and the proportional distribution of births in the stable population $\left\{{\underset{q}{1}}_{1}\right\}$. For our earlier two-region illustration involving slovenia and the rest of Yugoslavia, we have the following numerical approximations for these values:

$$
r=0.006099 \quad\left\{{\underset{\sim}{v}}_{1}(0)\right\}^{\prime}=\left[\begin{array}{lll}
0.357351 & 0.642649
\end{array}\right]
$$


$\underset{\sim}{\kappa}=\left[\begin{array}{rr}27.309929 & 0.016457 \\ 0.244865 & 27.104288\end{array}\right] \quad\left\{\underset{\sim}{\left.\mathbb{q}_{1}\right\}}=\left[\begin{array}{l}0.046478 \\ 0.953522\end{array}\right]\right.$

Substituting into (21) gives

$$
\{\underset{\sim}{Q}\}=\left[\begin{array}{c}
9,374 \\
192,304
\end{array}\right]
$$

a result that can be verified by calculating the regional stable equivalent populations $\{\underset{\sim}{Y}\}$, defined in Rogers (1975), and then using the relation (Willekens, 1977):

$$
\begin{aligned}
& \{\underset{\sim}{Q}\}=\underset{\sim}{b}\{\underset{\sim}{Y}\} \\
& =\left[\int_{0}^{\infty} e^{-r x} \underset{\sim}{m}(x) \underset{\sim}{\ell}(x) d x\right]\left[\int_{0}^{\infty} e^{-r x} \underset{\sim}{\ell}(x) d x\right]^{-1} \\
& =\left[\int_{0}^{\infty} e^{-r x} \underset{\sim}{\ell}(x) d x\right]^{-1} \underset{\sim}{\underset{\sim}{Y}\}} .
\end{aligned}
$$

The reader can verify that the earlier scalings of $\{\underset{\sim}{v}(0)\}^{\prime}$ and $\{\underset{\sim}{Q}\}$ found in section 3.2 give an identical numerical result for stable equivalent births.

\section{The Spatial Momentum of Zero Population Growth}

Differences between observed population age compositions and those of stationary populations make immediate zero growth an unlikely condition for most national populations. A closed population's birth rate and growth rate depend on its fertility schedule and its age composition. Consequently whether and how long a population continues to grow after achieving a net 
reproduction rate of unity depends on that population's age composition and its degree of divergence from that of a stationary population. The ratio by which the ultimate stationary population exceeds a current population is the momentum of that population.

4.1 The Ultimate Size and Distribution of a Stationary Spatial Population

If fertility were to drop immediately to replacement level in a population that is closed to migration, the ultimate stationary number of births in the resulting zero growth population would be (Keyfitz, 1975)

$$
\hat{\mathrm{Q}}=\frac{1}{\mu} \int_{0}^{\infty} \mathrm{k}(\mathrm{x}) \hat{\mathrm{v}}(\mathrm{x}) \mathrm{dx}=\frac{\hat{\mathrm{v}}}{\mu},
$$

where $\mu$ is the mean age of childbearing in the stationary population, and $\hat{v}(x)$ is the reproductive value corresponding to an intrinsic rate of growth $r=0$, a condition we can ensure by reducing fertility to replacement level along the lines described in section 2 of this paper. The corresponding ultimate stationary total population may be found by dividing $\hat{Q}$ by the stationary birth rate $\hat{b}$ or, equivalently, by multiplying it by $e(0)$, the expectation of life at birth

$$
\hat{Y}=\frac{\hat{Q}}{\hat{b}}=e(0) \hat{Q} \text {. }
$$

Such a calculation gives the same result as a full population projection carried out with the modified fertility schedule $\hat{\mathrm{m}}(\mathrm{a})$.

The above results have their spatial (multiregional) counterparts. To develop these it is convenient first to recall (18) and to define $\{\underset{\sim}{\hat{v}}(x)\}$ ' to be the vector of spatial reproductive values corresponding to an intrinsic rate of growth $r=0$. (We have seen earlier that a transition to zero 
growth may be carried out by multiplying the fertility schedule $\underset{\sim}{\mathrm{m}}(\mathrm{a})$ by the fertility adjustment matrix $\underset{\sim}{\gamma}$. .) Then the ultimate number of stationary equivalent births must be

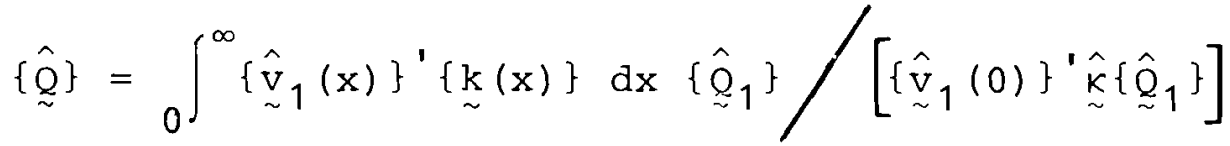

$$
\begin{aligned}
& =\frac{1}{\left\{\hat{\sim}_{1}(0)\right\}^{1} \underset{\sim}{\mu}\left\{\hat{\sim}_{1}\right\}} \hat{v}\left\{{\underset{\sim}{Q}}_{1}\right\}=\frac{\hat{v}}{\mu}\left\{{\underset{\sim}{Q}}_{1}\right\} \quad,
\end{aligned}
$$

where $\left\{\hat{\sim}_{1}(0)\right\}^{\prime}$ and $\left\{\hat{\sim}_{1}\right\}$ are, respectively, the left and right characteristic vectors associated with the unit dominant characteristic root of $\underset{\sim}{\gamma R}(0)$, and where

$$
\underset{\sim}{\mu}=\underset{\sim}{\hat{\kappa}}=\underset{\sim}{\gamma} R(1) \underset{\sim}{R}{ }^{-1}(0){\underset{\sim}{\gamma}}^{-1}
$$

is the matrix of mean ages of childbearing in the stationary population that evolves after the decline of fertility to replacement level.

The ultimate total stationary population is

$$
\{\underset{\sim}{\hat{\mathbf{Y}}}\}={\underset{\sim}{\hat{b}}}^{-1}\{\underset{\sim}{\hat{Q}}\}=\underset{\sim}{e}(0)\{\underset{\sim}{\hat{O}}\},
$$

where

$$
\underset{\sim}{\hat{b}}=\left[\int_{0}^{\infty} \hat{\sim}(a) \underset{\sim}{\ell}(a) d a\right]\left[\int_{0}^{\infty} \underset{\sim}{\ell}(a) d a\right]^{-1}=\underset{\sim}{\gamma} \underset{\sim}{R}(0){\underset{\sim}{e}}^{-1}(0),
$$

and $\underset{\sim}{e}(0)$ is a matrix of expectations of life at birth disaggregated by regions of birth and residence.

Equation (25) has a simple and intuitively appealing interpretation. Consistent with (23) it defines the total size of stationary equivalent births in a multiregional population to be equal to the quotient of the total reproductive value $\hat{v}$ and the weighted index $\mu=\left\{{\underset{\sim}{\hat{v}}}_{1}(0)\right\}{\underset{\sim}{\mu}}_{\sim}^{\left.\underset{Q_{1}}{\hat{q}_{1}}\right\}}$ in that population both evaluated after the decline in fertility to replacement 
level, and distributes that total according to the proportional allocation determined by the right characteristic vector associated with the unit dominant characteristic root of the modified net reproduction rate matrix $\underset{\sim}{\underset{\sim}{R}(0)}$. The interpretation of equation (26) follows in a straightforward manner.

4.2 The Spatial Momentum of an Initially Stable Population An abrupt decline in fertility to bare replacement level in a single-region population that initially is experiencing stable growth leads to a computationally simpler form for Equation (23). Keyfitz (1975) shows that in such an instance the ratio of the ultimate stationary population $\hat{Y}$ to the stable population of $\mathrm{K}$ individuals just prior to the decline in fertility is

$$
\frac{\hat{Y}}{K}=\frac{b e(0)}{\mu r} \quad\left(\frac{R(0)-1}{R(0)}\right),
$$

where $b$ is the birth rate, $r$ the rate of growth, e(0) the expectation of $\mathrm{life}$ at birth, and $\mathrm{R}(0)$ the net reproduction rate, all measured before the drop in fertility, and $\mu$ is the mean age of childbearing afterward. Expressing (27) as

$$
\hat{\mathrm{Y}}=e(0) \hat{Q}
$$

gives

$$
\hat{Q}=\frac{b K}{\mu r}\left(\frac{R(0)-1}{R(0)}\right)=\frac{1}{\mu r}[\gamma(R(0)-1) \Omega],
$$

where $\gamma=1 / R(0)$ and $Q=b K$. Note that this formula for total stationary equivalent births does not require the calculation of the total reproductive value of the population; but by virtue of (23) it implies that, in this special situation,

$$
\hat{\mathrm{v}}=\frac{\gamma}{\mathrm{r}}(\mathrm{R}(0)-1) \mathrm{Q} .
$$


Conventional methods of population projection may be used to obtain the future population that evolves from any particular observed or hypothetical regime of growth. Therefore (27) is not needed to obtain a numerical estimate of an ultimate stationary population. However keyfitz's simple momentum formula gives us an understanding of the population dynamics that are hidden in the arithmetical computations of a population projection. It identifies in an unambiguous way the five parameters of a current population that determine the size of the future population.

An analogous simplification of Equation (25) may be obtained in the multiregional model. If $\{k(x)\}$ is stable, then entering

$$
\left.\{\underset{\sim}{k}(x)\}=e^{-r x} \underset{\sim}{\ell}(x) \underset{\sim}{Q}\right\}
$$

into (25), and multiplying by $\underset{\sim}{ }(0)$ to produce the stationary equivalent population instead of stationary equivalent births, gives

$$
\begin{aligned}
& \{\underset{\sim}{\hat{Y}}\}=\frac{1}{\mu} \underset{\sim}{e}(0) \int_{0}^{\infty}\left\{{\underset{\sim}{v}}_{1}(x)\right\}^{\prime} e^{-r x} \underset{\sim}{\ell}(x)\{\underset{\sim}{Q}\} \quad d x \quad\left\{\underset{\sim}{\hat{Q}_{1}}\right\} \\
& =\frac{1}{\mu} \underset{\sim}{\mathrm{e}}(0)\left[\left\{{\underset{\sim}{\hat{v}}}_{1}(0)\right\}^{\prime} \underset{\sim}{\underset{\gamma}{\gamma}} \int^{\infty} \int^{\infty} \mathrm{e}^{-r \mathrm{x}} \underset{\sim}{\mathrm{m}}(\mathrm{a}) \underset{\sim}{\ell}(\mathrm{a}) \operatorname{dadx}\{\underset{\sim}{Q}\}\right]\left\{\underset{\sim}{\hat{Q}_{1}}\right\} .
\end{aligned}
$$

Evaluating the double integral and simplifying, we obtain

$$
\begin{aligned}
& \{\underset{\sim}{\hat{Y}}\}=\underset{\sim}{e}(0) \frac{1}{\mu r}\left[\left\{{\underset{\sim}{\hat{V}}}_{1}(0)\right\}^{\prime} \underset{\sim}{\underset{\sim}{\gamma}[\underset{\sim}{R}(0)-\underset{\sim}{\Psi}(r)]}\{\underset{\sim}{Q}\}\right]\left\{\hat{\sim}_{1}\right\} \\
& =\underset{\sim}{e}(0) \hat{Q}\left\{{\underset{\sim}{Q}}_{1}\right\},
\end{aligned}
$$

where

$$
\hat{Q}=\frac{1}{\mu r}\left[\left\{\hat{\sim}_{\sim}(0)\right\}^{\prime} \underset{\sim}{\gamma}[\underset{\sim}{R}(0)-\underset{\sim}{\Psi}(r)] \quad\{\underset{\sim}{Q}\}\right] .
$$


As with Equation (29), this formula does not recilire the calculation of the total reproductive value of the population, but it implies that

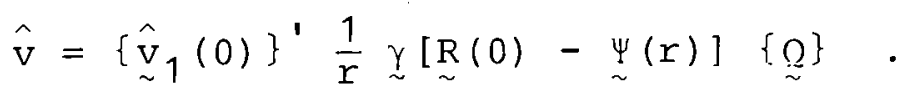

Equation (31) is not as practically useful as its singleregion counterpart because it is much more difficult to come up with accurate guesses or estimates of the values taken on by the many parameters. Thus a more effective procedure may be to first estimate the ultimate size of the total stationary equivalent births $\hat{Q}$, by means of (29); then to distribute that total among the various regions according to the allocation defined by the characteristic vector associated with the unit root of $\underset{\sim}{\gamma R}(0)$; and, finally, to premultiply the resulting vector by $e_{\sim}(0)$ to find $\{\hat{\sim}\}$. We now shall iliustrate such a procedure with a numerical example using data for India.

\subsection{The Urbanization Momentum in India}

We have shown that the geographical distribution of a spatial zero growth population depends very fundamentally on three matrices: $\underset{\sim}{e}(0), \underset{\sim}{R}(0)$, and $\underset{\sim}{\gamma}$. The first describes the multiregional levels of mortality and migration; the second sets out the multiregional net reproduction patterns before the decline in fertility; and the third defines the particular "spatial path" by which fertility is reduced. The product $\underset{\sim}{\gamma} R(0)$ gives $\underset{\sim}{\hat{R}}(0)$, whose characteristic vector associated with the unit root and scaled to sum to $\hat{Q}$ is $\{\hat{Q}\}$.

Equation (32) also may be used to illustrate dramatically that where people choose to live in the future presents issues and problems that are potentially as serious as those posed by the number of children they choose to have. Consider, for example, the projection to zero growth of India's population that was recently carried out by Norman Ryder on the basis of the following assumptions: 
To simplify the task of projecting the population of India, we make the following assumptions: it is a stable population with a growth rate $r=+0.025$ and survival functions corresponding to those labelled "West, level 13" (for which the female and male expectations of life at birth are 50 and 47.114, respectively) in the coale/ Demeny collection; the mean age of (gross) maternity $\mathrm{m}=29$; the ratio of male to female births $k=1.05$; and the current population size is 600 million. (Ryder, 1974, p. 6)

From these assumptions it follows that the initial number of female births per annum $\mathrm{B}(\mathrm{t})=12.156$ million, $\mathrm{R}(0)=2.019$, and $\mu=28.672$. Applying (28), Ryder finds a $\hat{0}$ of 8.558 million for females and a zero growth total (males plus females) population of approximately 851 million. He then shows that if survival levels in India eventually rise to e $(0)=70$ years for females and $e(0)=66.023$ for males, and

if replacement level fertility takes 40 years to achieve and the mean age of gross reproduction declines from 29 to 27 , the ultimate female birth cohort size will be..15.029 million. Given that value, ...the consequent ultimate population size is 2.094 billion. (Ryder, 1974, p. 7)

Ryder concludes that the thought of a population of 2.1 billion for India is staggering and goes on to examine in what respects the components of his projection may be modifiable.

There is no question but that the thought of a 2.1 billion population for India is staggering. What is even more mindboggling, however, is that anywhere from one to two thirds of this total is likely to eventually be found in that nation's already teeming and over-congested urban areas (the current figure is 20 percent). To show this, we need only to introduce a few additional assumptions and then apply Equation (32). Specifically, assume that life expectancy in India today is 57 years in urban areas and 48 years in rural areas, with the 
migration pattern being such that ${ }^{5}$

$$
\underset{\sim}{e(0)}=\left[\begin{array}{cc}
u^{e} u^{(0)} & r^{e}{ }^{(0)} \\
u^{e}{ }_{r}(0) & r^{e}{ }^{(0)}
\end{array}\right]=\left[\begin{array}{ll}
43 & 9 \\
12 & 39
\end{array}\right] \text {. }
$$

Assume, further, that the spatial pattern of net reproduction is given by ${ }^{6}$

$$
\underset{\sim}{R}(0)=\left[\begin{array}{ll}
1.10 & 0.25 \\
0.50 & 1.55
\end{array}\right] \text {. }
$$

Then an immediate decline in fertility to bare replacement level according to the cohort replacement alternative gives

$$
\underset{\sim}{\hat{R}(0)}=\left[\begin{array}{ll}
0.73 & 0.17 \\
0.27 & 0.83
\end{array}\right] \quad\left\{\hat{\sim}_{1}\right\}=\left[\begin{array}{l}
0.38 \\
0.62
\end{array}\right]
$$

5 According to a recent census publication (Registrar General of India, 1972), the crude death rates for urban and rural areas in India in 1970 were 10.2 and 17.3 per thousand, respectively. These were disaggregated into age-specific death rates using the age profiles and population data reported in this census publication and in the U.N. Demographic Yearbook (U.N., 1975). The crude outmigration rates for urban and rural areas in 1970 were set at 10.0 and 6.8, per thousand, respectively (Bose, 1973). A model migration age profile (Rogers, 1976) was used to disaggregate these into agespecific outmigration rates. A two-region life table, calculated using these age-specific mortality and migration rates, produced the matrix of regional expectations of life at birth set out above.

${ }^{6}$ Crude urban and rural birth rates in India in 1970 were 29.7 and 38.8 , per thousand, respectively. These were disaggregated into age-specific rates using the fertility age profile set out in Ambannavar (1975). The age-specific rates then were combined with the stationary population of the two-region life table referred to in footnote 5 to obtain the above matrix of net reproduction rates. 
an ultimate spatial zero growth population distribution of

$$
\{\underset{\sim}{\hat{Y}}\}=\left[\begin{array}{c}
369 \\
482
\end{array}\right] \text { million. }
$$

Under this projection, approximately 43 percent of the national population will be urban, giving rise to an urbanization momentum of roughly $1.42 \times(.43 / .20)=3.1$. A rough estimate of the corresponding momentum under Ryder's gradual fertility reduction alternative is $3.49 \times(.43 / .20)=7.6$.

The redistributional consequences of the proportional reduction alternative are quite different, however. In this case

$$
\underset{\sim}{\hat{\mathrm{R}}(0)}=\left[\begin{array}{ll}
0.63 & 0.14 \\
0.29 & 0.89
\end{array}\right] \quad\left\{{\underset{\sim}{\hat{Q}}}_{1}\right\}=\left[\begin{array}{l}
0.28 \\
0.72
\end{array}\right],
$$

if fertility declines immediately, and the spatial zero growth population that results is

$$
\{\underset{\sim}{\hat{\hat{y}}}\}=\left[\begin{array}{c}
315 \\
536
\end{array}\right] \quad \text { million. }
$$

Here only about a third (37 percent) of the zero growth population is urban. The rough estimates of the immediate and gradual fertility reduction momenta now become 2.6 and 6.5 , respectively. 7

7 The two fertility reduction alternatives differ not only in their redistributional impacts but also with respect to the urban and rural age compositions that they generate. The proportional reduction alternative produces an urban population that is over five years older in mean age than the corresponding rural population ( 38.3 to 33.0 years). The same difference in the cohort replacement alternative is not as pronounced, although the urban population is still older than the rural population in mean age ( 36.9 to 33.6 years). 
But rural-to-urban migration in India is surely going to increase in the course of its development and modernization. For example, urban-rural demographic data for the Soviet Union give approximately the following regional expectations of life at birth (Rogers, 1976):

$$
\underset{\sim}{e}(0)=\left[\begin{array}{ll}
60 & 41 \\
10 & 29
\end{array}\right]
$$

and the net reproduction matrix

$$
\underset{\sim}{R}(0)=\left[\begin{array}{ll}
0.80 & 0.64 \\
0.23 & 0.46
\end{array}\right] \text {. }
$$

By way of contrast, a very crude approximation of the corresponding net reproduction rate matrix for the U.S.A. is (Rogers and Willekens, 1975):

$$
\underset{\sim}{R}(0)=\left[\begin{array}{ll}
0.85 & 0.45 \\
0.25 & 0.90
\end{array}\right] \text {. }
$$

These data suggest that reasonable assumptions for India's future life expectancy matrix and its reduced net reproduction rate matrix (after, say a cohort replacement fertility decline) might be

$$
\underset{\sim}{e}(0)=\left[\begin{array}{ll}
59 & 23 \\
9 & 45
\end{array}\right] \text { and } \underset{\sim}{\hat{R}(0)}=\left[\begin{array}{cc}
3 / 4 & 1 / 3 \\
1 / 4 & 2 / 3
\end{array}\right] \text {. }
$$

The dominant characteristic vector $\left\{{\underset{\sim}{Q}}_{1}\right\}$ associated with the unit root of $\underset{\sim}{\hat{R}}(0)$ now is 


$$
\left\{{\underset{\sim}{Q_{1}}}_{1}\right\}=\left[\begin{array}{l}
0.57 \\
0.43
\end{array}\right],
$$

and this implies the spatial zero growth population distribu$\underline{\text { tion }}$

$$
\left\{{\underset{\sim}{\hat{Y}}}_{1}\right\}=\left[\begin{array}{l}
0.64 \\
0.36
\end{array}\right] \text {. }
$$

Under these very gross assumptions, approximately 64 percent of India's zero growth population would become urban, yielding an urbanization momentum of $1.42 \mathrm{x}(.64 / .20)=4.5$ for Ryder's case of immediate fertility reduction, and a corresponding momentum of $3.49 \times(.64 / .20)=11.2$ for the case of gradual fertility reduction. Figure 1 illustrates the various urbanization momenta calculated above for India and shows graphically the wide range of potential levels of urbanization for that country. Considering the variety of human settlement problems that already plague cities such as Calcutta, Bombay, and Delhi, it is a foreboding view of the future.

\section{Conclusion}

In this paper we have illustrated that migration and redistribution may present growth issues and problems that are potentially as serious as those posed by fertility and natural increase. This troublesome feature of spatial population dynamics appears even in zero growth populations, where the redistributional consequences of an immediate reduction of fertility levels can be of considerable importance.

With respect to methodological issues, this paper has demonstrated that the mathematical apparatus commonly used by demographers to examine the evolution of national populations to zero growth may be extended for application in spatial 
population analysis. The principal role in this extension is played by the characteristic matrix $\psi(r)$ and its right and left characteristic vectors, $\{\underset{\sim}{Q}\}$ and $\{\underset{\sim}{v}(0)\} '$, respectively. The former defines the regional allocation of stable equivalent births; the latter gives the spatial distribution of regional reproductive values at birth. This distinction is hidden in the single-region model, where stable equivalent births and the reproductive value at birth are cancelled out in each of their respective definitional equations to give $1=\psi(r)$. 
㲾

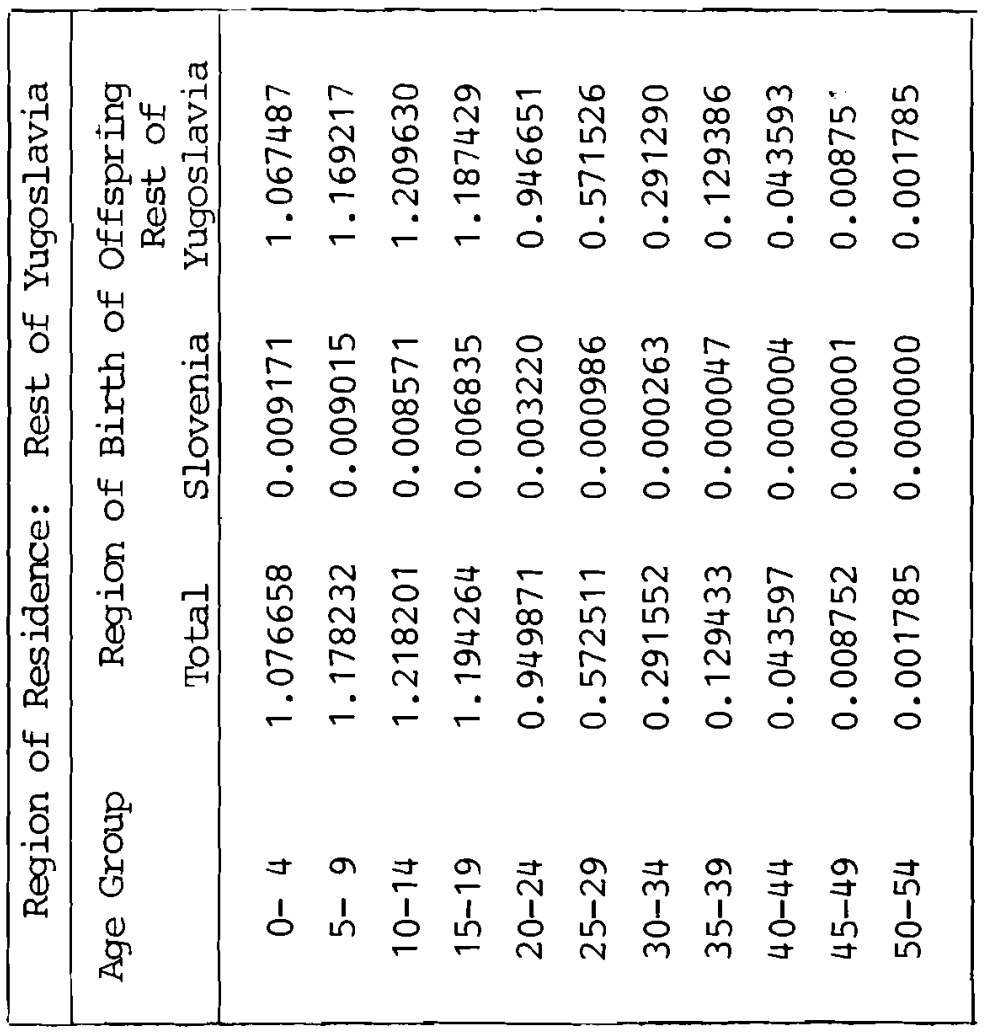

(1) ते

要

요

E $x$

잉

is

(1)

$+\sqrt{6}$

G 0

هి

प

戞?

4 15

З U O

च.

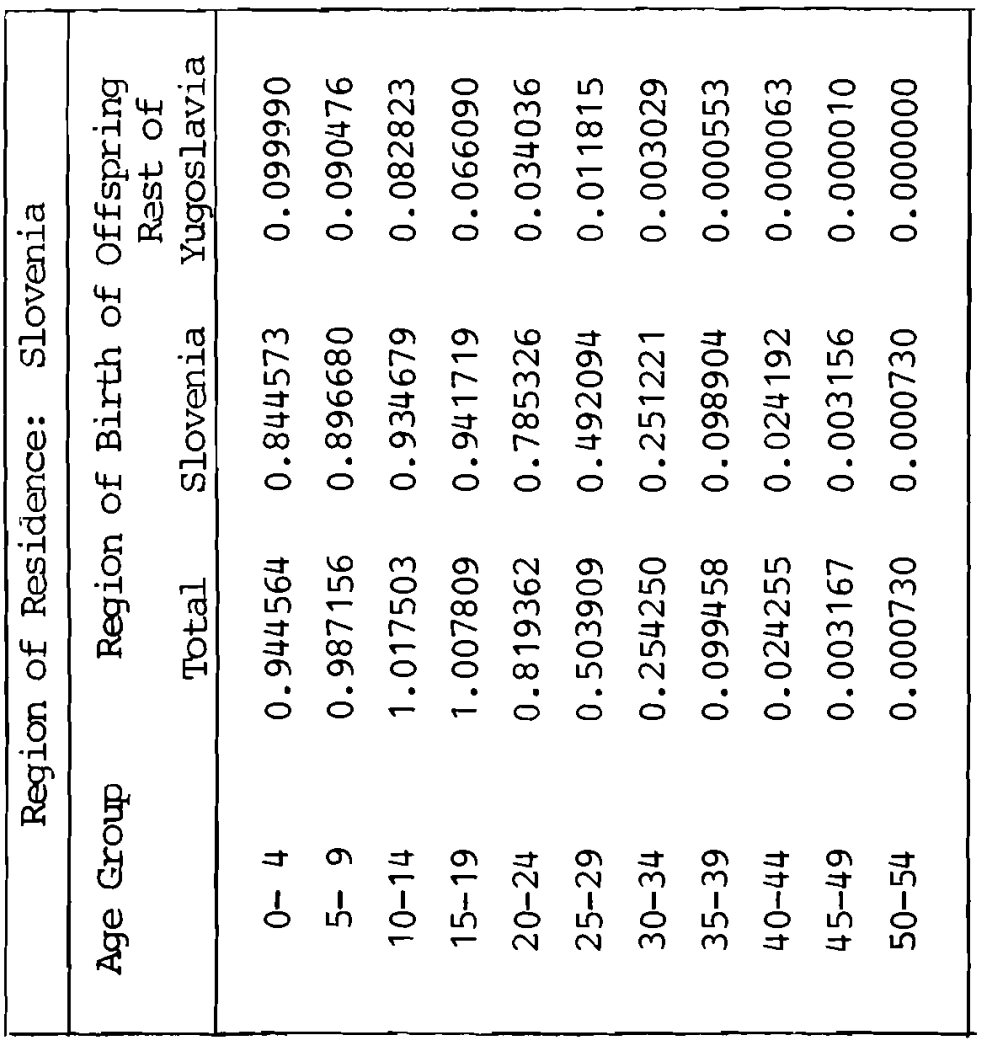


Table 2. Spatial reproductive value of females aged $\mathbf{x}$ to $x+4$ at last birthday, by region of residence: Yugoslavia, 1961.

\begin{tabular}{|ccc|}
\hline & \multicolumn{2}{c|}{ Region of Residence } \\
Age Group & Slovenia & Rest of Yugoslavia \\
\hline $0-4$ & 1.024392 & 1.928907 \\
$5-9$ & 1.059390 & 2.111699 \\
$10-14$ & 1.083626 & 2.183932 \\
$15-19$ & 1.060574 & 2.142272 \\
$20-24$ & 0.846536 & 1.705649 \\
$25-29$ & 0.513342 & 1.028800 \\
$30-34$ & 0.256669 & 0.524109 \\
$35-39$ & 0.099899 & 0.232731 \\
$40-44$ & 0.024305 & 0.078400 \\
$45-49$ & 0.003175 & 0.015738 \\
$50-54$ & 0.000730 & 0.003210 \\
\hline
\end{tabular}

Table 3. Total discounted number of daughters to observed female population by region of birth and residence: Yugoslavia, 1961.

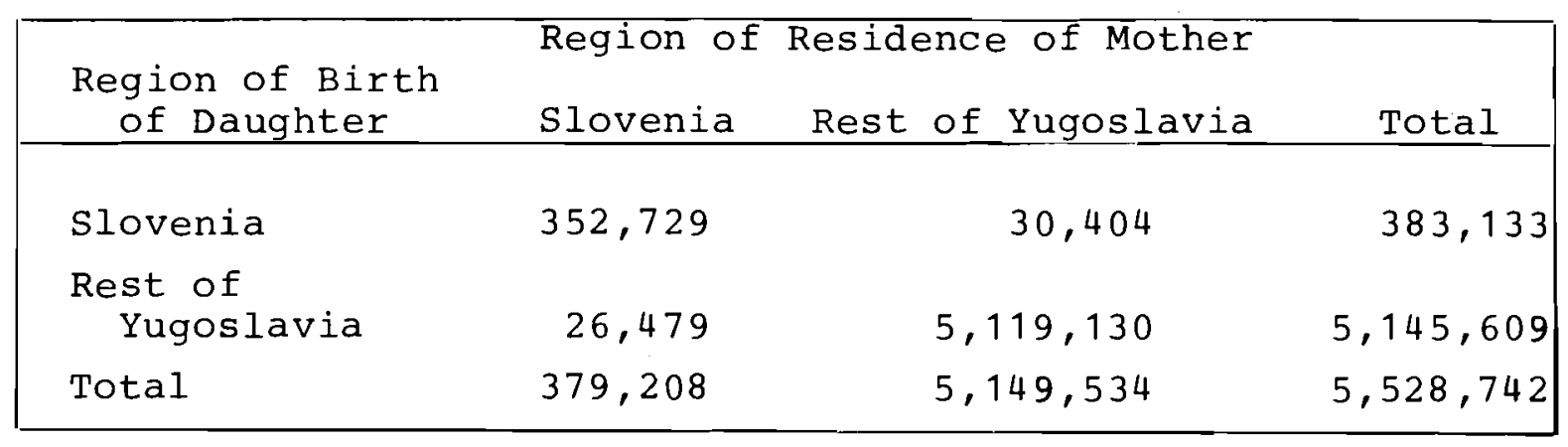




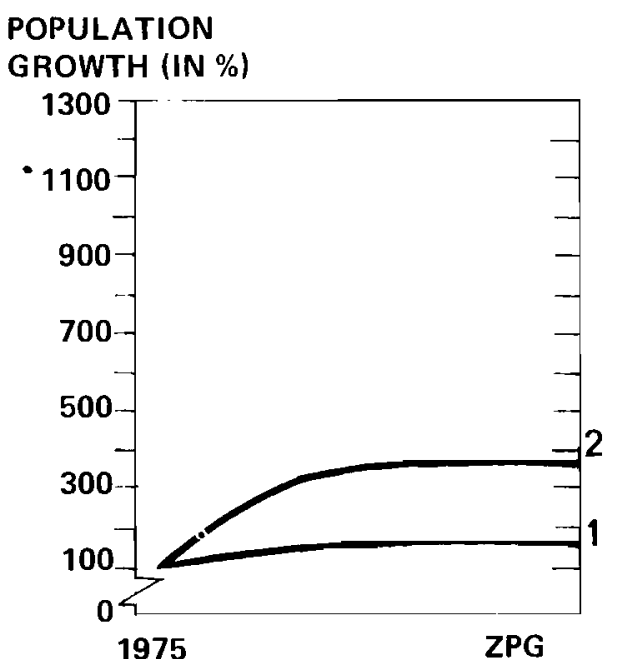

Key

1. Immediate Decline

2. Gradual Decline

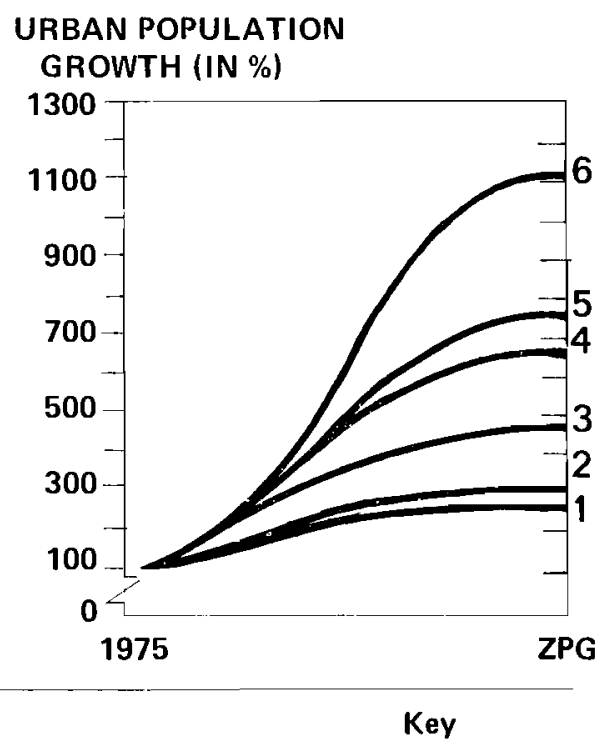

1. Immediate Proportional Reduction Alternative

2. Immediate Cohort Replacement Alternative

3. Immediate Cohort Replacement Alternative with Increased Mobility

4. Gradual Proportional Reduction Alternative

5. Gradual Cohort Replacement Alternative

6. Gradual Cohort Replacement Alternative with Increased Mobility

Figure 1. Urbanization momenta of India's population. 


\section{$\underline{\text { References }}$}

Ambannavar, J.P. (1975), Second India Studies: Population, The Macmillan Co. of India, Ltd., Delhi.

Bose, A. (1973), Studies in India's Urbanization 1901-1971, Tata McGraw-Hill Co., Bombay.

Fisher, R.A. (1929), The Genetical Theory of Natural Selection, 2nd rev. ed., Dover, New York.

Goodman, L. (1969), The Analysis of Population Growth when the Birth and Death Rates Depend Upon Several Factors, Biometrics, 25, 659-681.

Keyfitz, N. (1975), Reproductive Value: With Applications to Migration, Contraception, and Zero Population Growth, in H. Blalock et al., eds., Quantitative Sociology, Academic Press, New York, pp. 587-612.

Registrar General of India (1972), Pocket Book of Population Statisties, Census Centenary 1972, Registrar General and Census Commissioner, New Delhi, India.

Rogers, A. (1975), Introduction to Multipegional Mathematical Demography, John Wiley, New York.

(1976), Two Methodological Notes on Spatial Population Dynamics in the Soviet Union, RM-76-48, International Institute for Applied Systems Analysis, Laxenburg, Austria.

and F. Willekens (1975), Spatial Population

- Dynamies, RR-75-24, International Institute for Applied Systems Analysis, Laxenburg, Austria.

Ryder, N.B. (1974), Notes on stationary Populations, Population Index, XL, 1, 3-28.

United Nations (1975), Demographic Yearbook, United Nations, New York.

Willekens, F. (1977), The Spatial Reproductive Value: Theory and Applications, , International Institute for Applied Systems Analysis, Laxenburg, Austria, forthcoming. 
Papers of the Migration and Settlement Study

I. Papers in the DYNAMJCS Series

1. Andrei Rogers and Frans Wiljekens, "Spatial Population Dynamics," Rik-75...24, July, 1975, forthcoming in Papers, Regional Soience Association, Vol. 36, 1976.

2. Andrei Rogers and Jacques Ledent, "Multiregional Population Projection, "Introral working paper, August, 1975, in optimization Tecimiques: Modeling and Optimization in tie service of Man, Part 1, ed. Jean Cea, Springer-veriag, Berlin, 1976, pp. 31-58.

3. Andrei Rogers and Jacques Ledent, "Increment-Decrement Life Tables: A Comment, "internal working paper, October, 1975. phbjshed in Demography, 13, 2 (1976), $287-290$.

4. Andrei Rogers, "Spatiai Migration Expectancies," RM-75-57, November, 1975.

5. Andrei Rogers, "Aggregation and Decomposition in Population Projection," PM-76-11, May, 1976, published in revised form in Envirorment and Planning A, $\underline{8}$, (1976), 515-541.

6. Andrei Rogers and Luis J. Castro, "Model Multiregional Life Tables and Scable Populations," RR-76-09, MaY, 1976 .

7. Andrei Rogers ard Frans Willekens, "Spatial Zero Population Growth," RM-76-20, Apri1, 1976.

8. Frans willekens, "Sensitivty Analysis," RM-76-49, June, 1976 .

9. Andrei Rogers and Frans willekens, "The Spatial Reproductive Value and the spatial Momentum of Zero Population Growth," RM-76-. , December, 1976.

II. Papers in the DEMOMETRICS serjes

1. Andrei Rogers, "Demuretrics of Migration and Settlement," RM-76-68, August, 1976 .

2. John Miron, "Job-Search, Migration and Metropolitan Growth," RM-76-00, forthooining.

III. Papers in the POLICX Bnalysis Series

1. Yuri Evtushenko and Ross 13. Mackinnon, "Non-Linear Programming Approaches to rational set.tement systems

Planning," RR-75-25: July, 1975. 
2. R.K. Mehra, "An Optimal Control Approach to National Settlement Systems Planning," RM-75-58, November, 1975 .

3. Frans Willekens, "Optimal Migration Policies," RM-76-50, June, 1976.

4. Anatoli Propoi and Frans Willekens, "A Dynamic Linear Programming Approach to National Settlement Systems Planning," , forthcoming.

IV. Papers in the COMPARATIVE STUDY Series

1. Ross D. Mackinnon and Anna Maria Skarke, "Exploratory Analyses of the 1966-1971 Austrian Migration Table," RR-75-31, September, 1975.

2. Galina Kiseleva, "The Influence of Urbanization on the Birthrate and Mortality Rate for Major Cities in the U.S.S.R.," RM-75-68, December, 1975.

3. George Demko, "Soviet Population Policy," RM-75-74, December, 1975.

4. Andrei Rogers, "The Comparative Migration and Settlement Study: A Summary of Workshop Proceedings and Conclusions," RM-76-01, January, 1976.

5. Andrei Rogers, "Two Methodological Notes on Spatial Population Dynamics in the Soviet Union," RM-76-48, June, 1976.

6. Frans Willekens and Andrei Rogers, "Computer Programs for Spatial Demographic Analysis," RM-76-58, August, 1976 . 